\title{
可见光驱使铜盐催化芳香烃二氟烷基化反应
}

\author{
戴建玲雷文龙刘强* \\ (兰州大学功能有机分子化学国家重点实验室 化学化工学院 兰州 730000)
}

\begin{abstract}
摘要 以 $\mathrm{CuI}$ 为铜源, 通过原位形成光催化剂的途径, 实现了室温下可见光驱使铜催化澳二氟乙酸乙酯、澳二氟酰胺 等对芳烃及杂芳烃的二氟烷基化反应. 该反应条件温和、原料廉价易得、底物适用范围广、产率较高, 为合成二氟烷 基(杂)芳烃化合物提供了一种方法. 机理研究表明, 该反应可能经历了单电子转移的自由基反应历程.

关键词＼cjkstart烷基自由基; 可见光催化; 二氟烷基化反应; 芳烃; 铜
\end{abstract}

\section{Visible-Light-Driven Difluoroalkylation of Aromatics Catalyzed by Copper}

\author{
Dai, Jianling Lei, Wenlong Liu, Qiang* \\ (State Key Laboratory of Applied Organic Chemistry, Lanzhou University, Lanzhou 730000)
}

\begin{abstract}
The introduction of difluoromethyl groups into organic molecules not only can dramatically alter physical properties of nonfluorinated counterparts, but also provide valuable $\mathrm{CF}_{2}$-containing building blocks for the synthesis of other difluoromethylenated compounds. Therefore, there is a growing demand to develop efficient and practical methods for the introduction of the difluoromethyl motif. Although significant advances have been made in the preparation of difluoromethylated arenes, these reactions usually required pre-functionalized substrates, precious metal catalysts, elevated temperature, and so on. In the past decade, visible light-driven photoredox catalysis has been proved to be powerful in synthetic radical chemistry. Particularly, direct difluoroalkylations of arenes have been achieved using precious-metal photocatalysts such as ruthenium or iridium polypyridyl complexes. Herein, we are committed to developing a cheap copper-based phororedox system for direct difluoroalkylation of arenes. The key to this approach is the in-situ formation of cuprous photocatalyst from cuprous iodide, an imine ligand (2,9-dichloro-1,10-phenanthroline) and a triaryl phosphine ligand (4,5-bis(diphenylphosphino)-9,9-dimethyl xanthene). With catalytic amount of reagents mentioned above, the direct difluoroalkylation between arenes and difluoroalkylation reagents $\left(\mathrm{BrCF}_{2} \mathrm{CO}_{2} \mathrm{Et}\right.$ or $\left.\mathrm{BrCF}_{2} \mathrm{CONR}^{1} \mathrm{R}^{2}\right)$ took place smoothly under $6 \mathrm{~W}$ blue LED irradiation at room temperature. A variety of electron-rich arenes, including electron-donating aromatics, indoles, furans, thiophenes, and pyrimidines, could be carbonyldifluoromethylated in moderate to excellent yields. In addition, high yields were obtained for the intramolecular and intermolecular aminocarbonyldifluoromethylation by the catalytic system. Preliminary mechanistic studies reveal that $[\mathrm{Cu}(\mathrm{dcp})(\mathrm{xantphos})] \mathrm{I}(\mathrm{dcp}=2,9$-dichloro-1,10-phenanthroline, xantphos $=4,5$-bis (diphenyl phosphino)-9,9-dimethyl xanthene), in situ-formed from CuI, dcp, and xantphos should be the real photocatalyst to catalyze the visible light-driven difluoroalkylation. Difluormethyl radicals, produced by single electron transfer from the excited photocatalyst to difluoroalkylation reagents, should be involved in the difluoroalkylation. In summary, visible-light driven difluoroalkylation of arenes with difluoroalkylation reagents via $\mathrm{Cu}$-catalysis has been developed. The use of the bidentate phosphine ligand and the imine ligand is essential for high efficiency as they could bind to cuprous iodide to generate the photocatalyst in situ. The typical procedure is as follows: a mixture of arenes $(0.6 \mathrm{mmol}), \mathrm{CuI}(0.02 \mathrm{mmol}), \mathrm{dcp}(0.02 \mathrm{mmol})$, xantphos $(0.02 \mathrm{mmol}), \mathrm{K}_{3} \mathrm{PO}_{4}(0.4 \mathrm{mmol})$ and $\mathrm{CH}_{2} \mathrm{Cl}_{2}(2 \mathrm{~mL})$ were loaded in a flame-dried reaction vial which was subjected to evacuation with argon for $30 \mathrm{~min}$. Subsequently, $\mathrm{BrCF}_{2} \mathrm{CO}_{2} \mathrm{Et}(0.2 \mathrm{mmol})$ was added to the mixture via syringe, and the mixture continued degassing for $5 \mathrm{~min}$. After degassing procedure, the vial was sealed with wax, and irradiated by blue light for $24 \mathrm{~h}$. The reaction was monitored by TLC. Further purification of the evaporated mixture by flash column chromatography on silica gel (eluent: petroleum ether/ethyl acetate) gave the desired product.

Keywords alkyl radical; visible-light photocatalysis; difluoroalkylation; arenes; copper
\end{abstract}

\section{1 引言}

含氟类化合物具有很好的亲脂性、代谢稳定性和生 物利用度 ${ }^{[1]}$, 在农药、医药和生命科学等领域中有非常
重要的应用价值 ${ }^{[2]}$. 据相关报道, 超过 $20 \%$ 的药物和 $30 \%$ 的农用化学品至少含有一个氟源 ${ }^{[3]}$, 畅销的氟化药 物包括抗抑有药氟西汀(Prozac $)^{[4]}$ 、降胆固醇药阿托伐他 汀(Lipitor) ${ }^{[5]}$ 和抗菌环丙沙星(Ciprobay) ${ }^{[6]}$. 在众多含氟

\footnotetext{
*E-mail: liuqiang@1zu.edu.cn

Received May 15, 2019; published August 14, 2019.
}

Supporting information for this article is available free of charge via the Internet at http://sioc-journal.cn.

Project supported by the National Natural Science Foundation of China (Nos. 21572090 and 21871123) and the Fundamental Research Funds for the Central Universities (lzujbky-2017-k05).

项目受国家自然科学基金(Nos. 21572090 and 21871123)和兰州大学中央高校基础研究基金(lzujbky-2017-k05)资助. 
有机化合物中, 含二氟烷基取代的芳烃化合物受到了越 来越多的关注 ${ }^{[7]}$. 二氟亚甲基通常被认为是氧和羰基的 等极体 ${ }^{[8]}$, 引入二氟亚甲基能够增强其邻位基团的酸 性、改变分子的偶极以及产生构象的变化 ${ }^{[9]}$. 早在上个 世纪，黄维垣等 ${ }^{[10]}$ 实现了全氟卤代烷烃在保险粉参与 下的亚磺化脱卤反应. 近年来, 过渡金属钯 ${ }^{[11]} 、 \mathrm{c}^{[12]}$ 、 铜 ${ }^{[13]}$ 等催化(杂)芳香烃二氟烷基化反应研究取得了重要 进展，官能团化的二氟烷基化试剂 $\mathrm{BrCF}_{2} \mathrm{CO}_{2} \mathrm{Et}$ 、 $\mathrm{BrCF}_{2} \mathrm{C}(\mathrm{O}) \mathrm{NHR}$ 等被广泛用于实现各种芳烃和杂芳烃 的二氟烷基化反应. 通常, 这类反应需要较高的反应温 度克服 $\mathrm{C}-\mathrm{H}$ 活化的能垒. 随着可见光催化的蓬勃发 展 ${ }^{[14]}$, 室温下通过可见光照射实现(杂)芳香烃 $\mathrm{C}-\mathrm{H}$ 键 二氟烷基化反应成为可能. Qing ${ }^{[15 a, 15 b]}$ 、 Wang ${ }^{[15 c]}$ 、 $\mathrm{Cho}^{[15 \mathrm{~d}]} 、$ Stephenson ${ }^{[15 \mathrm{e}]}$ 课题组等先后报道了可见光催化 条件下(杂)芳烃二氟烷基化. 我们课题组 ${ }^{[15 \mathrm{f}, 15 \mathrm{~g}]}$ 也实现 了 $\mathrm{BrCF}_{2} \mathrm{C}(\mathrm{O}) \mathrm{NHR}$ 和 $\mathrm{BrCF}_{2} \mathrm{PO}(\mathrm{OR})_{2}$ 等对(杂)芳烃的分 子间及分子内二氟烷基化反应. 然而, 这些反应均使用 贵金属钌(II)或铱(III)配合物为光催化剂. 因此, 发展廉 价金属催化且室温条件下可高效发生的(杂)芳烃二氟烷 基化方法十分必要.

和钌(II)或铱(III)配合物相比, 铜盐配合物作为光催 化的研究非常有限 ${ }^{[16]}$. 其中, 二邻菲咯啉铜(I)配合物 $\left[\mathrm{Cu}(\text { dap })_{2}\right] \mathrm{Cl}(\text { dap }=2,9 \text {-bis(4-anisyl)-1,10-phenanthroline })^{[17]}$ 和邻菲咯啉双膦铜(I)配合物 ${ }^{[18]}$ 为常见的铜(I)配合物光 催化剂. 和 $\left[\mathrm{Cu}(\mathrm{dap})_{2}\right] \mathrm{Cl}$ 相比, 邻菲咯啉双膦铜(I)配合 物激发态寿命更长, 且易于通过膦配体和氮配体取代基 的双重调控实现期望的激发态性能 ${ }^{[18 \mathrm{c}, 18 \mathrm{~d}, 18 \mathrm{~h}]}$. 近年来 Collins $^{[19]} 、 \mathrm{Fu}$ 和 Peters ${ }^{[20]}$ 和 Wang ${ }^{[21]}$ 小组的研究表明, 廉 价的亚铜盐可在邻菲咯啉及双膦配体存在下原位生成 邻菲咯啉双膦铜(I)光催化剂, 实现一系列有趣的可见光 催化反应. 因此, 廉价亚铜盐通过邻菲咯啉及双膦配体 的篮选, 有可能原位生成具备合适光氧化还原 (photo-redox) 能力的邻菲咯啉双膦铜(I)光催化剂, 从而 催化可见光照射下(杂)芳烃的二氟烷基化反应.

\section{2 结果与讨论}

首先研究了均三甲氧基苯和溴二氟乙酸乙酯在不 同条件下的二氟烷基化反应. 均三甲氧基苯与溴二氟乙 酸乙酯在含有 $\mathrm{CuI}(10 \mathrm{~mol} \%) 、 2,9$-二甲基菲咯啉(10 $\mathrm{mol} \%$ )、4,5-双(二苯基膦)-9,9-二甲基氧杂葱(10 $\mathrm{mol} \%$ ) 的二氯甲烷 $(2 \mathrm{~mL})$ 溶液中, 以磷酸钾 (2.0 equiv.)作为碱, 氩气环境中室温下光照(6W LED 蓝灯为光源) $24 \mathrm{~h}$ 后, 目标产物 $3 \mathbf{a}$ 的收率为 $87 \%$ (表 1, Entry 1). 接下来我们 考察了不同的铜源, 如 $\mathrm{Cu}\left(\mathrm{CH}_{3} \mathrm{CN}\right)_{4} \mathrm{PF}_{6} 、 \mathrm{Cu}(\mathrm{OAc})_{2}$ 等, 但产率并未得到提升(表 1, Entries 4,5). 随后对 $\mathrm{K}_{2} \mathrm{CO}_{3}$ 、 $\mathrm{KOAc}$ 等不同的碱进行笁选, 结果表明 $\mathrm{K}_{3} \mathrm{PO}_{4}$ 是合适的 碱. 将溶剂 $\mathrm{CH}_{2} \mathrm{Cl}_{2}$ 用 $\mathrm{CH}_{3} \mathrm{OH} 、 \mathrm{DMF}$ 和 $\mathrm{CH}_{3} \mathrm{CN}$ 等替代 后, 产物 3a 的产率均明显降低(表 1, Entries 8 10), 因
表 1 亚铜盐催化溴二氟乙酸乙酯对均三甲氧基苯的二氟烷基化反应 条件优化

Table 1 Optimization of conditions for copper catalyzed difluoromethylation of trimethoxybenzene with $\mathrm{BrCF}_{2} \mathrm{CO}_{2} \mathrm{Et}$

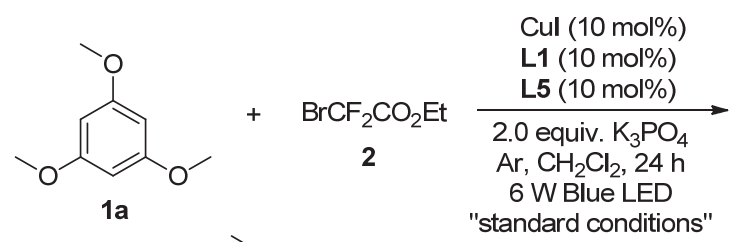<smiles>CCOC(=O)C(F)(F)F</smiles>

$3 \mathbf{a}$

\begin{tabular}{|c|c|c|}
\hline Entry & Changes from the "standard conditions" & Yield $^{a}$ \\
\hline 1 & No changes & 87 \\
\hline 2 & Without CuI, $\mathrm{K}_{3} \mathrm{PO}_{4}$, or $\mathbf{L 5}$ & 0 \\
\hline 3 & Without L1 & 40 \\
\hline 4 & $\mathrm{Cu}(\mathrm{OAc})_{2}$ instead of $\mathrm{CuI}$ & 0 \\
\hline 5 & $\mathrm{Cu}(\mathrm{MeCN})_{4} \mathrm{PF}_{6}$ instead of $\mathrm{CuI}$ & 85 \\
\hline 6 & KOAc instead of $\mathrm{K}_{3} \mathrm{PO}_{4}$ & 77 \\
\hline 7 & $\mathrm{~K}_{2} \mathrm{CO}_{3}$ instead of $\mathrm{K}_{3} \mathrm{PO}_{4}$ & 86 \\
\hline 8 & $\mathrm{CH}_{3} \mathrm{CN}$ instead of DCM & 61 \\
\hline 9 & $\mathrm{CH}_{3} \mathrm{OH}$ instead of DCM & 21 \\
\hline 10 & DMF instead of DCM & 40 \\
\hline 11 & 2.0 equiv. $1 \mathrm{a}$ & 80 \\
\hline 12 & $\mathbf{L} 2$ instead of $\mathbf{L} 1$ & 95 \\
\hline 13 & $\mathbf{L 3}$ instead of $\mathbf{L} 1$ & 0 \\
\hline 14 & L4 instead of L1 & 0 \\
\hline 15 & L6 instead of L5 & 0 \\
\hline 16 & {$[\mathrm{Cu}(\mathrm{dcp})(\mathrm{xantphos})] \mathrm{I}(10 \mathrm{~mol} \%)$} & 92 \\
\hline 17 & $\mathrm{CuI}(5 \mathrm{~mol} \%)$, L2 (5 mol $\%)$ and $\mathbf{L 5}(5 \mathrm{~mol} \%)$ & 88 \\
\hline 18 & 1.0 equiv. $\mathrm{K}_{3} \mathrm{PO}_{4}$ & 30 \\
\hline 19 & under air (open flask) & 0 \\
\hline 20 & dark & 0 \\
\hline
\end{tabular}

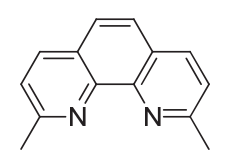

L1, dmp

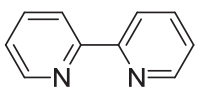

L4, bpy

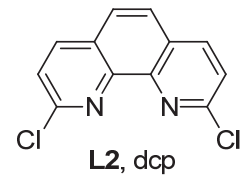

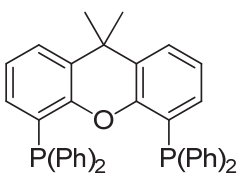

L5, xantphos

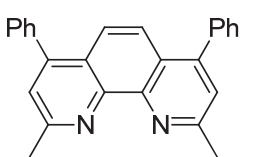

L3, bc

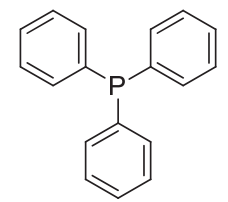

L6, $\mathrm{PPh}_{3}$

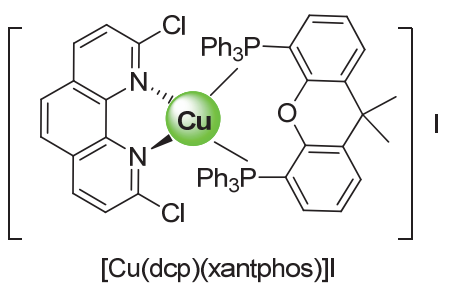

a Yields were determined by ${ }^{1} \mathrm{H}$ NMR. $\mathrm{dmp}=$ neocuproine, $\mathrm{dcp}=$ 2,9-dichloro-1,10-phenanthroline, $b c=$ bathocuproin, $b p y=2,2^{\prime}$-bipyridine, xantphos $=4,5$-bis(diphenylphosphino)-9,9-dimethylxanthene, $\mathrm{PPh}_{3}=$ triphenylphosphane. 
此 $\mathrm{CH}_{2} \mathrm{Cl}_{2}$ 是反应的理想溶剂. 在确定反应最佳亚铜盐、 碱和溶剂后, 我们进一步耖选了反应配体(表 1, Entries 12 15), 发现当氮配体为 2,9-二氯-1,10-菲咯啉(dcp)、 双膦配体为 4,5-双 (二苯基膦)-9,9-二甲基氧杂葱 (xantphos), 产率提升到 95\%. 此外, 我们还尝试了降低 催化剂、配体以及碱的用量(表 1 , Entries 17, 18), 发现当 CuI、dcp 和 xantphos 的量降低到 $5 \mathrm{~mol} \%$ 时，仍有 $88 \%$ 产率的二氟烷基化均三甲氧基苯 $\mathbf{3 a}$ 生成. 最后, 控制实 验证明反应必须在绝氧和可见光照射下方可发生.

在优化反应条件下, 我们研究了该可见光催化二氟 烷基化反应底物的适用性和官能团的兼容性. 如表 2 所 示, 大量芳烃化合物能与溴二氟乙酸乙酯反应得到良好 到优秀的产率( $54 \% \sim 95 \%)$. 并且, 富电子芳烃产率较

表 2 碘化亚铜催化澳二氟乙酸乙酯对芳烃的二氟烷基化反应 ${ }^{a}$

Table 2 Difluoromethylation of aromatics with $\mathrm{BrCF}_{2} \mathrm{CO}_{2} \mathrm{Et}$ catalyzed by cuprous iodide

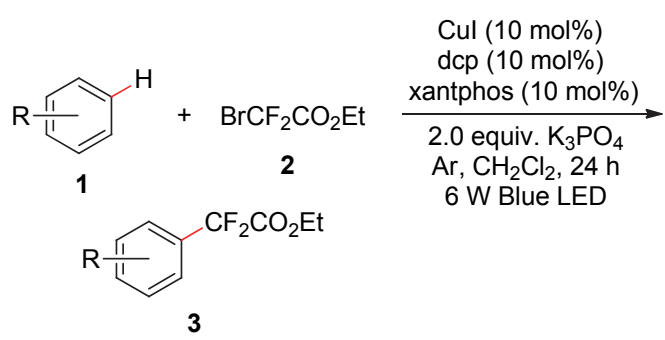

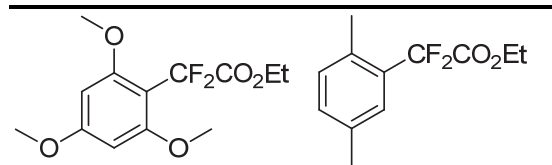

3a, $95 \%$<smiles>CCOC(=O)C(F)(F)c1ccccc1OC</smiles>

$3 d, 65 \%$<smiles>CCOC(=O)C(F)(F)c1cc(C=O)ccc1C(C)C</smiles>

$3 g, 54 \%$

3b, $84 \%$<smiles>CCOC(=O)C(F)(F)c1cc(C)ccc1OC</smiles>

$3 e, 63 \%$<smiles>CCOC(=O)C(F)(F)c1ccccc1-c1ccccc1</smiles>
3h, 78\% $o: p=2: 1$<smiles>CCOC(=O)C(F)(F)c1cccc(I)c1</smiles>

$3 \mathbf{k}, 88 \%$ $o: m: p=1: 1: 1$

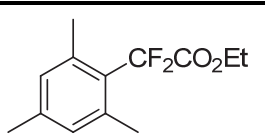

3c, $80 \%$<smiles>CCOC(=O)C(F)(F)c1c(OC)cc(Br)cc1OC</smiles>

3f, $66 \%$<smiles>CCOC(F)(F)c1ccc(N(C)C)cc1</smiles>

3i, $75 \%$ o:p $=1: 6$<smiles>CCOC(=O)C(F)(F)c1ccccc1N(c1ccccc1)c1ccccc1</smiles>

3j, $83 \%$ o:p: $m^{b}=7: 6: 1$
${ }^{a}$ Reaction conditions: 1 (0.60 mmol, 3.0 equiv. $), 2(0.20 \mathrm{mmol}), \mathrm{CuI}(0.02$ $\mathrm{mmol})$, dcp (0.02 mmol), xantphos (0.02 mmol), $\mathrm{K}_{3} \mathrm{PO}_{4}(0.4 \mathrm{mmol})$ in $\mathrm{CH}_{2} \mathrm{Cl}_{2}$ $(2.0 \mathrm{~mL})$ were irradiated with $6 \mathrm{~W}$ blue LED for $24 \mathrm{~h}$ at r.t. Isolated yield. ${ }^{b} o=$ ortho-position, $m=$ meta-position, $p=$ para-position.
高，位点选择性与预期的自由基对芳基取代途径一致. 例如，当含有不同取代基的芳烃存在时 $(\mathbf{3 h}, \mathbf{3 j} \sim 3 \mathbf{3})$ ，相 应官能团化的二氟甲基化产物为异构体的混合物. 且由 于二氟烷基自由基具有一定的亲电性，电子云密度较大 的芳香烃位点更易于发生二氟烷基化反应( $\mathbf{3 i}, 3 \mathbf{3} \sim \mathbf{3 g})$. 进一步地，探究了最优反应条件对杂芳烃的二氟烷基化 (表 3). 结果表明吡啶、吲哚、嘧啶、呋喃、噻吩等芳杂 环对该反应体系均兼容，并取得良好到优秀的产率 (58\% 95\%). 接下来，研究了 $N, N$-二甲基苯胺和溴二 氟酰胺的反应(表 $4, \mathbf{6 a} \sim \mathbf{6 c}$ )，结果表明该反应体系可以 以 $38 \% \sim 70 \%$ 的产率实现不同溴二氟酰胺对 $N, N$-二甲 基苯胺的二氟烷基化. 最后，又进一步尝试了分子内的 酰胺化反应，得到较高的产率(68\% 70\%).

表 3 碘化亚铜催化溴二氟乙酸乙酯对杂芳烃的二氟烷基化反应 ${ }^{a}$ Table 3 Difluoromethylation of hetero aromatics with $\mathrm{BrCF}_{2} \mathrm{CO}_{2} \mathrm{Et}$ catalyzed by cuprous iodide

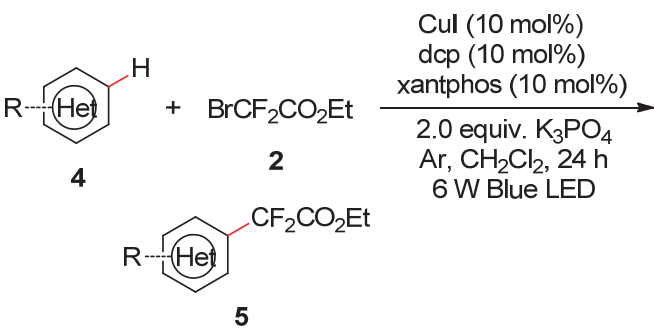

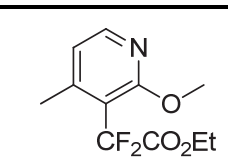

5a, $58 \%$

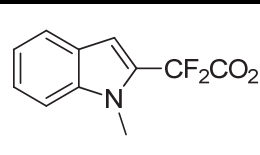

$5 \mathbf{b}, 60 \%$

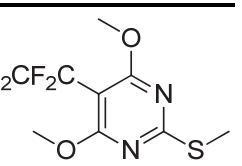

5c, $95 \%$<smiles>CCOC(F)(F)Cc1c(C)nc(C(C)C)nc1O</smiles>

$5 d, 78 \%$<smiles>CCOC(=O)Cc1cc2ccccc2o1</smiles>

o: $m^{b}=10: 1$<smiles>CCOC(=O)C(F)(F)c1sccc1OC</smiles>

$5 f, 65 \%$
${ }^{a}$ Reaction conditions: 4 (0.60 mmol, 3.0 equiv.), 2 (0.20 mmol), $\mathrm{CuI}(0.02$ mmol), dep (0.02 mmol), xantphos $(0.02 \mathrm{mmol}), \mathrm{K}_{3} \mathrm{PO}_{4}(0.4 \mathrm{mmol})$ in $\mathrm{CH}_{2} \mathrm{Cl}_{2}$ $(2.0 \mathrm{~mL})$ were irradiated with $6 \mathrm{~W}$ blue LED for $24 \mathrm{~h}$ at r.t. Isolated yield. ${ }^{b}{ }_{o}=$ ortho-position, $m=$ meta-position

为了进一步理解催化剂碘化亚铜及配体在该可见 光催化体系中的角色，进行了一系列控制实验. 首先, 在光反应中, 若缺少菲咯啉配体 $\mathrm{dcp}$ 或双膦配体 xantphos，反应均不能很好地进行(表 1, Entries 2,3)，说 明亚铜盐是和菲咯啉配体及双膦配体三组分原位形成 配合物并充当光催化剂. 随后，使用事先制备好的 $[\mathrm{Cu}(\mathrm{dcp})(\mathrm{xantphos})] \mathrm{I}(10 \mathrm{~mol} \%)$ 替代碘化亚铜、菲咯啉配 体 dcp 和双膦配体 xantphos, 得到了与最优反应条件相 似催化效果(表 1, Entry 16). 并且, 原位生成的亚铜催化 剂 $(\mathrm{CuI} / \mathrm{dcp} /$ xantphos $=1: 1: 1)$ 的吸收光谱与 $[\mathrm{Cu}(\mathrm{dcp})$ (xantphos) $] \mathrm{I}$ 的吸收光谱一致(图 1). 因此, 该二 氟甲基化应该是由碘化亚铜、菲咯啉配体及双膦配体原 位生成配合物 $[\mathrm{Cu}(\mathrm{dcp})(\mathrm{xantphos})]$ 作为光催化剂, 进而 
被可见光激发驱动的.

表 4 碘化亚铜催化溴二氟乙酰胺的分子间及分子内反应 ${ }^{a}$

Table 4 Intermolecular and intramolecular reaction of bromodifluoroacetamide catalyzed by cuprous iodide<smiles>CN(C)c1ccccc1C(F)(F)F</smiles>

$6 a, 38 \%$

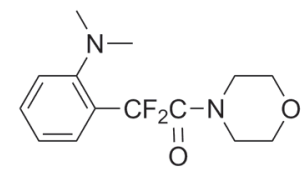

$6 c, 70 \%$<smiles>CN(C)c1ccccc1C(F)(F)F</smiles>

6b, $65 \%$<smiles>O=C1Nc2ccccc2C1(F)F</smiles>

$6 d, 68 \%$

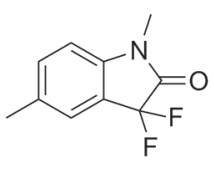

$6 e, 70 \%$
${ }^{a}$ Reaction conditions: $6(0.20 \mathrm{mmol}), \mathrm{CuI}(0.02 \mathrm{mmol}), \mathrm{dcp}(0.02 \mathrm{mmol})$, xantphos $(0.02 \mathrm{mmol}), \mathrm{K}_{3} \mathrm{PO}_{4}(0.4 \mathrm{mmol})$ in $\mathrm{CH}_{2} \mathrm{Cl}_{2}(2.0 \mathrm{~mL})$ were irradiated with $6 \mathrm{~W}$ blue LED for $24 \mathrm{~h}$ at r.t. Isolated yield.

在最优反应条件下, 当在该反应中加入过量的氮氧 自由基 2,2,6,6-四甲基哌啶-1-氧化物(TEMPO)或自由基 清除剂 2,6-二叔丁基羟基甲苯(BHT)后，均三甲基苯没 有产物 3c 生成. 结合表 2 中芳烃二氟烷基化反应可生成 苯环不同位置二氟烷基化异构体的事实, 可推测反应应 该通过生成二氟烷基自由基进行. 综合以上实验结果, 提出了反应的可能机理: 首先, 碘化亚铜和菲咯啉配体 及双膦配体原位形成邻菲咯啉双膦铜 (I) 配合物 $\left[\mathrm{Cu}^{\mathrm{I}} \mathrm{L}_{\mathrm{n}}\right]$. 该配合物经可见光照射被激发, 并和氟化试剂 $\mathrm{BrCF}_{2} \mathrm{CO}_{2} \mathrm{Et}$ 发生氧化淬灭产生 $\left[\mathrm{Cu}^{\mathrm{II}} \mathrm{L}_{\mathrm{n}}\right]$ 和二氟烷基自由 基 $\cdot \mathrm{CF}_{2} \mathrm{CO}_{2} \mathrm{Et}$. 该自由基加成到芳环上产生活性物种 $1 \mathbf{A}$, 由于芳构化的驱动力和强吸电子基团二氟乙酸乙
酯的诱导作用，该活性物质 $\mathbf{1 A}$ 的 $\alpha-\mathrm{H}$ 具有一定的酸 性 ${ }^{[22]}$, 使得 $\mathbf{1 A}$ 在 $\mathrm{K}_{3} \mathrm{PO}_{4}$ 作用下能有效地脱质子生成 $\mathbf{1 B}$, $1 B$ 经 $\left[\mathrm{Cu}^{\mathrm{II}} \mathrm{L}_{\mathrm{n}}\right]$ 氧化生成目标产物(图 2).

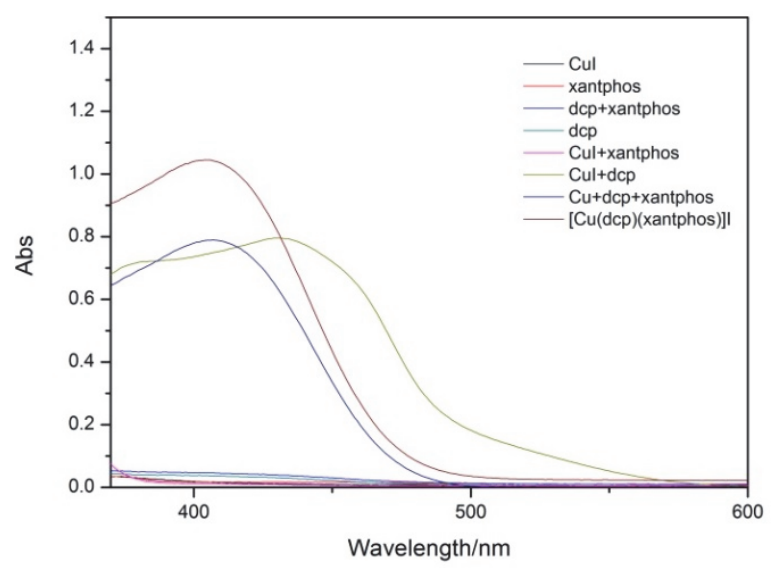

图 1 UV-vis 吸收光谱

Figure 1 UV-vis absorption spectra of substrates

\section{3 结论}

本文通过廉价亚铜盐和配体原位生成光催化剂的 策略, 实现了可见光驱使(杂)芳香烃和二氟烷基化试剂 $\left(\mathrm{BrCF}_{2} \mathrm{CO}_{2} \mathrm{Et}, \mathrm{BrCF}_{2} \mathrm{C}(\mathrm{O}) \mathrm{NHR}\right)$ 的分子间及分子内二氟 烷基化反应. 该反应条件温和、原料廉价易得、底物适 用范围广，具有潜在的应用价值.

\section{4 实验部分}

可见光催化下的芳烃二氟烷基化反应的实验方法: 向 $10 \mathrm{~mL}$ 干燥的硬质玻璃管中依次加入 $\mathrm{CuI}(0.02$

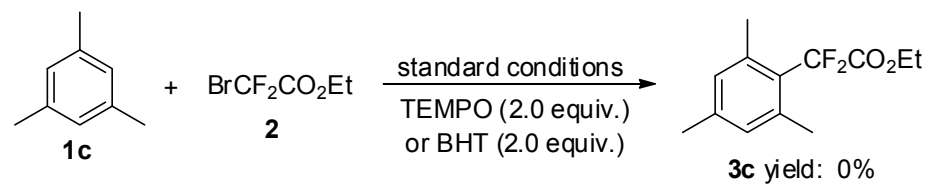

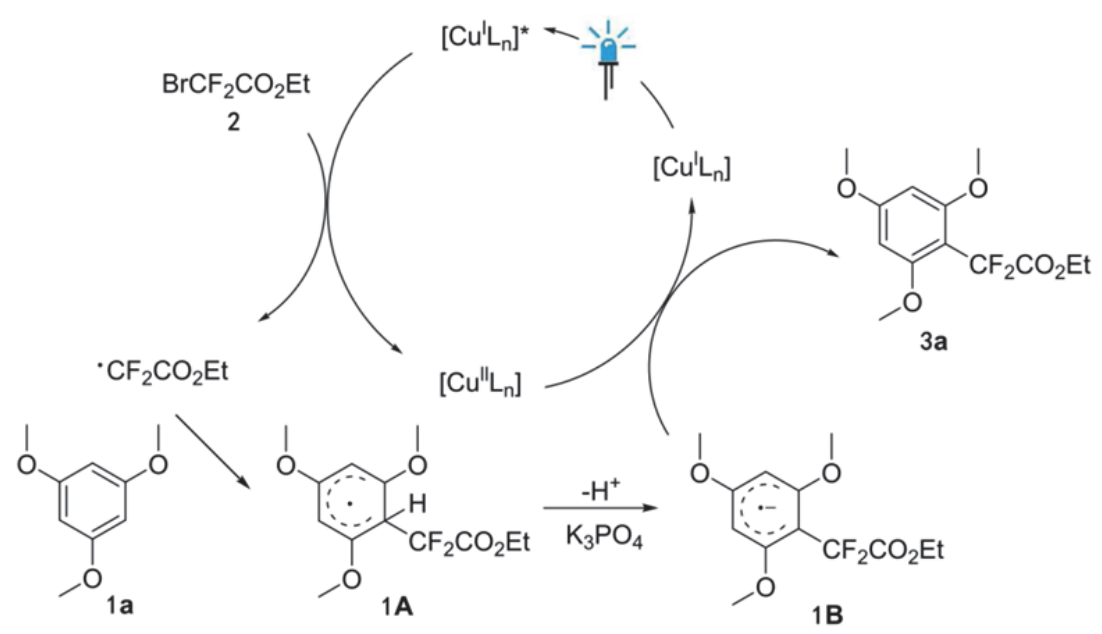

图 2 研究机理的控制实验以及可能的机理

Figure 2 Control experiment of mechanism studies and possible mechanism 
mmol $)$ dep $(0.02 \mathrm{mmol}) 、$ xantphos $(0.02 \mathrm{mmol})$ 、 $\mathrm{BrCF}_{2} \mathrm{CO}_{2} \mathrm{Et}(0.2 \mathrm{mmol}) 、 \mathrm{~K}_{3} \mathrm{PO}_{4}(2.0$ equiv.)和底物 $1 \mathrm{a}$ (0.6 mmol). 随后, 将玻璃管中的空气用氩气置换, 并在 氩气氛围下加入 $2.0 \mathrm{~mL}$ 二氯甲烷. 在玻璃管密封后, 将 其放在 $6 \mathrm{~W}$ 蓝光 LED 灯光反应中, 室温搅拌并照射 24 h. 等反应结束, 减压浓缩反应液后, 用 $200 \sim 300$ 目的 硅胶、石油醚/乙酸乙酯洗脱剂柱层析分离纯化产物. 目 标产物 3a 的产率为 $95 \%$.

\section{References}

[1] (a) Müller, K.; Faeh, C.; Diederich, F. Science 2007, 317, 1881. (b) Purser, S.; Moore, P. R.; Swallow, S.; Gouverneur, V. Chem. Soc. Rev. 2008, 37, 320 .

[2] (a) Jeschke, P. ChemBioChem 2004, 5, 570. (b) Purser, S.; Moore, P. R.; Swallow, S.; Gouverneur, V. Chem. Soc. Rev. 2008, 37, 320. (c) Wang, J.; Sanchez-Rosello, M.; del Pozo, C.; Sorochinsky, A. E.; Fustero, S.; Soloshonok, V. A.; Liu, H. Chem. Rev. 2014, 114, 2432.

[3] (a) Bégué, J. P.; Bonnet-Delpon, D. J. Fluorine Chem. 2006, 127, 992. (b) Isanbor, C. J. Fluorine Chem. 2006, 127, 303. (c) Kirk, K. L. J. Fluorine Chem. 2006, 127, 1013.

[4] Wong, D. T.; Bymaster, F. P.; Engleman, E. A. Life Sci. 1995, 57, 411.

[5] Roth, B. D. In Progress in Medicinal Chemistry, Vol. 40, Eds.: King, F. D.; Oxford, A. W., Elsevier, Amsterdam, 2002, pp. $1 \sim 22$.

[6] Drlica, K.; Malik, M. Curr. Top. Med. Chem. 2003, 3, 249.

[7] (a) Purser, S.; Moore, P.-R.; Swallow, S.; Gouverneur, V. Chem. Soc. Rev. 2008, 37, 320. (b) Nenajdenko, V. G.; Shastin, A. V. Chem. Rev. 2015, 115, 973. (c) Ni, C.-F.; Hu, J.-B. Chem. Rev. 2015, 115, 765. (d) Liang, T.; Ritter, T. Angew. Chem., Int. Ed. 2013, 52, 8214. (e) Zhou, B.-Y.; Cheng, J.-P. Org. Lett. 2016, 18, 6128. (f) Yu, W.; Qing, F.-L. Org. Lett. 2016, 18, 5130. (g) Guo, W.-H.; Zhang, X. ACS Catal. 2017, 7, 896. (h) Fu, X.-P.; Xiao, Y.-L.; Zhang, X. Chin. J. Chem. 2018, 36, 143. (i) He, X.; Gao, X.; Zhang, X. Chin. J. Chem. 2018, 36, 1059. (j) Fujiwara, Y. J.; Dixon, A.; Baran, P. S. J. Am. Chem. Soc. 2012, 134, 1494. (k) Xu, L.; Vicic, D. A. J. Am. Chem. Soc. 2016, 138, 2536. (1) Qi, Q.-Q.; Shen, Q.-L.; Lu, L. J. Am. Chem. Soc. 2012, 134, 6548. (m) Feng, Z.; Zhang, X. Org. Lett. 2016, 18, 44. (n) Ruan, Z.-X.; Zhang, S.-K.; Ackermann, L. Angew. Chem., Int. Ed. 2017, 56, 2045.

[8] (a) Blackburn, C. M.; England, D. A.; Kolkmann, F. J. Chem. Soc. Chem. Commun. 1981, 930. (b) Yang, Y.; You, Z.; Qing, F.-L. Acta Chim. Sinica 2012, 70, 2323 (in Chinese). (杨义, 游正伟, 卿凤悀, 化学学报, 2012, 70, 2323.) (c) An, L.; Tong, F.-F.; Zhang, X. Acta Chim. Sinica 2018, 76, 977 (in Chinese). (安伦, 童非非, 张新刚, 化学学报, 2018, 76, 977.)

[9] (a) Meanwell, N. A. J. Med. Chem. 2011, 54, 2529. (b) Meanwell, N. A. J. Med. Chem. 2018, 61, 5822 .

[10] (a) Huang, B. N.; Huang, W. Y.; Hu, C. M. Acta Chim. Sinica 1981, 39, 481 (in Chinese). (黄炳南, 黄维垣, 胡昌明, 化学学报, 1981, 39, 481.) (b) Huang, B. N.; Huang, W. Y.; Hu, C. M. J. Fluorine Chem. 1983, 23, 193. (c) Huang, B. N.; Huang, W. Y.; Wang, W. Acta Chim. Sinica 1983, 41, 1193 (in Chinese). (黄维垣, 黄炳南, 王巍，化学学报， 1983, 41, 1193.) (d) Wu, F.-H.; Huang, B. N.; Huang, W. Y. Chin. J. Org. Chem. 1993, 13, 449 (in Chinese). (吴范 宏, 黄炳南, 黄维垣, 有机化学, 1993, 13, 449.)

[11] Shi, S.-L.; Buchwald, S.-L. Angew. Chem. Int. Ed. 2017, 129, 2077.

[12] (a) Belhomme, M.-C.; Poisson, T.; Pannecoucke, X. J. Org. Chem. 2014, 79, 7205; (b) Wang, L.-P.; Liu, H.-Y.; Li, F.-F.; Zhao, J.-Q.;
Zhang, H.-Y.; Zhang, Y.-C. Adv. Synth. Catal. 2019, 361, 2354

[13] (a) Ruan, Z.-X.; Zhang, S.-K.; Zhu, C.-J.; Ruth, P.-N.; Stalke, D.; Ackermann, L. Angew. Chem., Int. Ed. 2017, 129, 2077; (b) Li, Z.-Y.; Li, L.; Li, Q.-L.; Jing, K.; Xu, H.; Wang, G.-W. Chem. Eur. J., 2017, 23, 3285. (c) Yuan, C.-C.; Chen, X.-L.; Zhang, J.-Y.; Zhao, Y.-S. Org. Chem. Front. 2017, 4, 1867.

[14] (a) Chen, Y.-Y.; Lu, L.-Q.; Yu, D.-G.; Zhu, C.-J.; Xiao, W.-J. Sci China Chem. 2019, 62, 24. (b) Liu, Q.; Wu, L.-Z. Nat. Sci. Rev. 2017, 4, 359. (d) Skubi, K. L.; Yoon, T. P. Nature 2014, 515, 45.

[15] (a) Lin, Q.; Chu, L.; Qing, F. Chin. J. Chem. 2013, 31, 885. (b) Yu, X.; Xu, X.-H.; Qing, F. Org. Lett. 2016, 18, 5130. (c) Su, Y.-M.; Hou, Y.; Yin, F.; Xu, Y.-M.; Li, Y.; Zheng, X.; Wang, X. Org. Lett. 2014, 16, 2958. (d) Jung, J.; Kim, E.; You, Y.; Cho, E. J. Adv. Synth. Catal. 2014, 356, 2741. (e) McAtee, R.-C.; Beatty, J.-W.; McAtee, C.-C.; Stephenson, C. R. J. Org. Lett. 2018, 20, 3491. (f) Wang, L.; Wei, X.-J.; Lei, W.-L.; Chen, H.; Wu, L.-Z.; Liu, Q. Chem. Commun. 2014, 50, 15916. (g) Wang, L.; Wei, X.-J.; Jia, W.-L.; Zhong, J.-J.; Wu, L.-Z.; Liu, Q. Org. Lett. 2014, 16, 5842.

[16] (a) Paria, S.; Reiser, O. ChemCatChem 2014, 6, 2477. (b) Reiser, O. Acc. Chem. Res. 2016, 49, 1990. (c) Hernandez-Perez, A. C.; Collins, S. K. Acc. Chem. Res. 2016, 49, 1557. (d) Cuttell, D. G.; Kuang, S.-M.; Fanwick, P. E.; McMillin, D. R.; Walton, R. J. Am. Chem. Soc. 2002, 124, 6. (e) McMillin, D. R.; McNett, K. M. Chem. Rev. 1998, 98, 1201. (f) Cuttell, D. G.; Kuang, S.-M.; Fanwick, P. E.; McMillin, D. R.; Walton, R. A. J. Am. Chem. Soc. 2002, 124, 6.

[17] (a) Huang, J.; Mara, M. W.; Stickrath, A. B.; Kokhan, O.; Harpham, M. R.; Haldrup, K.; Shelby, M. L.; Zhang, X.; Ruppert, R.; Sauvage, J.-P.; Chen, L. X. Dalton Trans. 2014, 43, 17615. (b) Pirtsch, M.; Paria, S.; Matsuno, T.; Isobe, H. T.; Reiser, O. Chem. Eur. J. 2012, 18, 7336. (c) Paria, S.; Pirtsch, M.; Kais, V.; Reiser, O. Synthesis 2013, 45, 2689. (d) Tang, X.-J.; Dolbier, W. R., Jr. Angew. Chem., Int. Ed. 2015, 54, 4246. (e) Bagal, D. B.; Kachkovskyi, G.; Knorn, M.; Rawner, T.; Bhanage, B. M.; Reiser, O. Angew. Chem., Int. Ed. 2015, 54, 6999. (f) Fumagalli, G.; Rabet, P. T. G.; Boyd, S.; Greaney, M. F. Angew. Chem., Int. Ed. 2015, 54, 11481. (g) Rabet, P. T. G.; Fumagalli, G.; Boyd, S.; Greaney, M. F. Org. Lett. 2016, 18, 1646. (h) Hossain, A.; Engl, S.; Lutsker, E.; Reiser, O. ACS Catal. 2019, 9, 1103.

[18] (a) Hernandez-Perez, A. C.; Vlassova, A.; Collins, S. K. Org. Lett. 2012, 14, 2988. (b) Knorn, M.; Rawner, T.; Czerwieniec, R.; Reiser, O. ACS Catal. 2015, 5, 5186. (c) Murat, A.-Z.; Hu, X.-L. Organometallics 2018, 37, 3928. (d) Brunner, F.; Graber, S.; Baumgartner Y.; Haussinger, D.; Prescimone, A.; Constable, E. C.; Housecroft, C. E. Dalton Trans. 2017, 46, 6379. (e) Nitelet, N.; Thevenet, D.; Schiavi, B.; Hardouin, C.; Fournier, J.; Tamion, R.; Pannecoucke, X.; Jubault, P.; Poisson, T. Chem. Eur. J. 2019, 25, 3262. (f) Wang, B.; Shelar, D. P.; Han, X.-Z.; Li, T.-T.; Guan, X.-G. Chem. Eur. J. 2015, 21, 1184. (g) Michelet, B.; Deldaele, C.; Kajouj, S.; Moucheron, C.; Evano, G. Org. Lett. 2017, 19, 3576. (h) Lu, W.; Liu, K.; Chen, Y.; Fu, W.-F.; Che, C.-M. Chem. Eur. J. 2015, 21, 1184 .

[19] (a) Hernandez-Perez, A. C.; Collins, S. K. Angew. Chem. Int. Ed. 2013, 52, 12696. (b) Hernandez-Perez, A. C.; Collins, S. K. Angew. Chem. Int. Ed. 2013, 125, 12928. (c) Hernandez-Perez, A. C.; Vlassova, A.; Collins, S. K. Org. Lett. 2012, 14, 2988.

[20] (a) Ahn, J. M.; Peters, J. C.; Fu, G. C. J. Am. Chem. Soc. 2017, 139, 18101. (b) Zhao, W.; Wurz, R. P.; Peters, J. C.; Fu, G. C. J. Am. Chem. Soc. 2017, 139, 12153.

[21] Wang, W.; Guo, M.-Z.; Qi, R.-P.; Shang, Q.-Y.; Liu, Q.; Wang, S.; Zhao, L.; Wang, R.; Xu, Z.-Q. Angew. Chem. Int. Ed. 2018, 57, 15841.

[22] Zhang, B.; Daniliuc, C. G.; Studer, A. Angew. Chem., Int. Ed. 2013, 52,10792 . 